\title{
Personalized Thermal Comfort Forecasting for Smart Buildings via Locally Weighted Regression with Adaptive Bandwidth
}

\author{
Carlo Manna, Nic Wilson and Kenneth N. Brown \\ Cork Constraint Computation Centre(4C), Dept. Computer Science, University College Cork, Cork, Ireland \\ \{c.manna,n.wilson\}@4c.ucc.ie,k.brown@cs.ucc.ie
}

\begin{abstract}
Keywords: Machine Learning, Smart Buildings, Thermal Comfort.
Abstract: A personalized thermal comfort prediction method is proposed for use in combination with smart controls for building automation. Occupant thermal comfort is traditionally measured and predicted by the Predicted Mean Vote (PMV) metric, which is based on extensive field trials linking reported comfort levels with the various factors. However, PMV is a statistical measure applying to large populations, and the actual thermal comfort could be significantly different from the predicted value for small groups of people. Moreover it may be hard to use for a real-time controller due to the number of sensor readings needed. In the present paper, we propose Robust Locally Weighted Regression with Adaptive Bandwidth (LRAB), a kernel based method, to learn individual occupant thermal comfort based on historical reports. Using publicly available datasets, we demonstrate that this technique is significantly more accurate in predicting individual comfort than PMV and other kernel methods. Therefore, is a promising technique to be used as input to adpative HVAC control systems.
\end{abstract}

\section{INTRODUCTION}

Energy-efficiency for buildings is currently a topic of major interest, especially with increasing energy costs. In particular, the HVAC (heating ventilation and air conditioning) system, which ensures thermal comfort conditions in offices (during both wintertime and summer), on the other is one of the main energy consumers in a building. For this reason, accurately predicting comfort levels for the occupants can enable one to avoid unnecessary heating or cooling, and thus improve the energy efficiency of the HVAC systems. A number of thermal comfort indices (indicators of human comfort) have been studied for the design of HVAC systems (Fanger, 1972; Gagge et al., 1986), the most widely used of which is the Predicted Mean Vote (PMV) index, which was developed by Fanger (Fanger, 1972). However, to find appropriate set-point temperatures with the PMV standard, the designer has to make assumptions about clothing and activity of occupants. Moreover, for small groups of people within a single room or zone in a building, PMV may not perform an accurate prediction as pointed out in (Kumar and Mahdavi, 2001) and in (Humphreys and Nicol, 2000). To address these issues, newer standards such as the recently accepted revisions to ASHRAE Standard 55, that include a new adaptive comfort standard (ACS) (de Dear and Brager, 2002), suggests an alternative (or a complementary) theory of thermal perception based on the psychological dimension of adaptation, which may be particularly important in contexts where people's interactions with the environment (i.e. personal thermal control), or diverse thermal experiences, may alter their expectations, and thus, their thermal sensation and satisfaction. In particular, the level of comfort perceived by each individual also depends on their degree of adaptation to the context and to the environmental changes, and therefore the specificity of each individual should be taken into account to learn and predict comfort satisfaction.

For these reasons, in the present paper, we propose an alternative approach tailored to individual occupants, which relies on historical data on individual responses to internal environment conditions. We propose Robust Locally Weighted Regression (Cleveland, 1979) with an Adaptive Bandwidth (LRAB), a kernel based method, to learn, automatically, the comfort model of each user based on their history. We applied this method using up to three input variables (inside air temperature, humidity and mean radiant temperature) which do not require expensive sensors and are easy to measure. Finally, we compare the proposed method with both PMV and a standard kernel 
method, the Nadaraya-Watson kernel method (with different kernel functions), using publicly available datasets (ASHRAE, RP-884). Our experimental results show that LRAB outperforms both the PMV and the Nadaraya-Watson kernel method in predicting individual comfort, and hence it is a promising technique to be used as an input to the heating/cooling control systems in an office environment.

The paper is organised as follows: in the next section, some background on PMV and on alternative existing techniques are reported. Then, in section 2 , the Nadaraya-Watson kernel and the proposed method are described, while in the section 3 , the experimental results using a public dataset are shown. Finally, in section 4 , conclusions and future directions are reported.

\subsection{Previous research}

Many methods have been proposed for comfort evaluation and prediction; a comprehensive overview is given in (Olesen, 2004). However, as stated in the previous section, the most widely used of these is the PMV index, which has been an international standard since the 1980s (ASHRAE, 2010), (ISO, 1994). The conventional PMV model predicts the mean thermal sensation vote on a standard scale for a large group of people in a given indoor climate. Like the other methods described in (Olesen, 2004), it is a function of two human variables and four environmental variables, i.e. clothing insulation worn by the occupants, human activity, air temperature, air relative humidity, air velocity and mean radiant temperature. The values of the PMV index have a range from -3 to +3 , which corresponds to an occupant's thermal sensation from cold to hot, with the zero value of PMV meaning neutral. As mentioned above, PMV is not just an index to measure the comfort level, but it is also, and especially, a model to predict the thermal sensation given the indoor environmental conditions. It has been validated by many studies, both in climate chambers and in buildings (Busch, 1992; Yang and Zhang, 2008). The standard approach to comfort-based control involves regulating the internal environment variables to ensure a PMV value of zero (Shukor et al., 2007; Yang and Su, 1997; Freire et al., 2008).

\subsubsection{Predicted mean vote and its alternative versions}

Although PMV can be succesfully used in a design phase (both for houses and buildings), it has some drawbacks when used for HVAC controllers. Firstly, it requires a substantial amount of environmental data. For a controller in real-time this information is only accessible via sensors. However some measurements, such as air velocity, require costly sensors, while, there are no sensors for variables such as clothing and activity level. Secondly, PMV is a statistical measure which assumes a large number of people experiencing the same conditions. For small groups of people within a single room or zone in a building, however, PMV may not be an accurate measure. (Kumar and Mahdavi, 2001) analysed the discrepancy between predicted mean vote proposed in (Fanger, 1972) and observed values based on a meta-analysis of the field studies database made available under ASHRAE RP-884 and finally proposing a framework to adjust the value of thermal comfort indices (a modified PMV). The large field studies on thermal comfort described in (Humphreys and Nicol, 2002), (de Dear and Schiller Brager, 2001) and (Humphreys and Nicol, 2000), have shown that PMV does not give correct predictions for all environments. (de Dear and Schiller Brager, 2001) found PMV to be unbiased when used to predict the preferred operative temperature in the air conditioned buildings. PMV did, however, overestimate the subjective warmth sensations of people in warm naturally ventilated buildings. (Humphreys and Nicol, 2000) showed that PMV was less closely correlated with the comfort votes than were the air temperature or the mean radiant temperature, and that the effects of errors in the measurement of PMV were not negligible. Finally the work in (Humphreys and Nicol, 2002) also showed that the discrepancy between PMV and the mean comfort vote was related to the mean temperature of the location. In addition to the relative inaccuracy, the PMV model is a nonlinear relation, and it requires iteratively computing the root of a nonlinear equation, which may take a long computation time. Therefore, a number of authors have proposed alternative methods of calculation to the main one proposed in (Fanger, 1972). Fanger and (ISO, 1994) suggest using tables to determine the PMV values of various combinations between the six thermal variables. (Sherman, 1985) proposed a simplified model to estimate the PMV value without any iteration step, by linearizing the radiation exchange term in Fanger's model. This study indicated that the simplified model was only accurate when the occupants are close to being comfortable. (Federspiel and Asada, 1992) proposed a thermal sensation index, which is a modified form of Fanger's model. They assumed that the radiative exchange and the heat transfer coefficient are linear, and they also assumed that the clothing insulation and heat generation rate of human activity are constant. They then derived a thermal sensation index that is an explicit function of the four environmental variables. However, as the authors said, the simplification of Fanger's 
PMV model results in significant error when the assumptions are not respected. On the other hand, in (Bingxin et al., 2011) and (Atthajariyakul and Leephakpreeda, 2005) different approaches have been proposed in order to compute PMV avoiding the difficult iterative calculation. The former proposes a Genetic Algorithm Back Propagation neural network to learn user comfort based both on historical data and realtime environmental measurements. The latter proposes a neural network applied to the iterative part of the PMV model that, after a learning phase, based on historical data, avoids the evaluation of such iterative calculation in real-time.

\subsubsection{Beyond the PMV}

Recent trends in the study of thermal environment conditions for human occupants are reported in the recently accepted revisions to ASHRAE Standard 55, which includes a new adaptive comfort standard (ACS). According to (de Dear and Brager, 2002) this adaptive model could be an alternative (or a complementary) theory of thermal perception. The fundamental assumption of this alternative point of view states that factors beyond fundamental physics and physiology play an important role in building occupants' expectations and thermal preferences. PMV does take into account the heat balance model with environmental and personal factors, and is able to account for some degrees of behavioral adaptation such as changing one's clothing or adjusting local air velocity.

However, it does not take into account the possible adaptation of an individual as a result from his/her interaction with the environment (i.e. personal thermal control), which, in turn, may alter his/her expectations, and thus, their thermal sensation and satisfaction. In particular, the level of comfort perceived by each individual also depends on their degree of adaptation to the context and to the environmental changes, and therefore the specificity of each individual should be taken into account to learn and predict comfort satisfaction.

For this reason, some authors have proposed techniques based on learning the perception of comfort by individuals. For example, in (Feldmeier and Paradiso, 2010) the author proposes a system able to learn individual thermal preferences using a Nearest Neighbor Classifier, taking into account only four variables (air temperature, humidity, clothing insulation and human activity), acquired by means of wearable sensors. In (Schumann et al., 2010), a Nearest Neighbor Classification-like method was implemented in order to learn individual user preferences based on historical data, using only one variable (air temperature). On other hand in (Daum et al., 2011) the authors propose a personalized measure of thermal comfort based on logistic regression to convert user votes to a probability of comfort.

In this study we consider such alternative and more practical approaches to predicting thermal comfort through the automatic learning of the comfort model of each user based on his/her historical records. We apply the Robust Locally Weighted Regression technique (Cleveland, 1979) with an Adaptive Bandwidth (LRAB), one of the family of statistical pattern recognition methods. Non-parametric regression methods, or kernel-based methods, are well established methods in statistical pattern recognition (Hastie et al., 2003). These methods do not need any specific prior relation among data. Hence, there are no parameter estimates in non-parametric regression. Instead, to forecast, these methods retain the data and search through them for past similar cases. This strength makes non-parametric regression a powerful method due to its flexible adaptation in a wide variety of situations. The Robust Locally Weighted Regression is one of a number of nonparametric regressions. It fits data by local polynomial regression and joins them together. The first version of this method was first introduced by Cleveland (Cleveland, 1979), and it was further developed for multivariate models in (Cleveland and Devlin, 1988).

\section{KERNEL METHODS}

In this section we describe a class of techniques used throughout this paper, which achieve flexibility in estimating the regression function $f(X)$ over domain $\mathfrak{R}^{p}$ by fitting a different but simple model separately at each query point $\mathbf{x}$ in $\mathfrak{R}^{p}$. This is done by using only those observations close to the target point $\mathbf{x}$ to fit the simple model, and in such a way that the resulting estimated function $\widehat{f(X)}$ is smooth. This localization is achieved by means of a weighting function kernel $\mathbf{K}_{\lambda}\left(\mathbf{x}, \mathbf{x}_{\mathbf{i}}\right)$, assigning a weight to $\mathbf{x}_{\mathbf{i}}$ based on its distance from $\mathbf{x}$. The kernels $\mathbf{K}_{\lambda}$ are generally characterized by a parameter $\lambda$ that is related to the width of the neighborhood (i.e. kernel bandwidth). In the next section we first describe the Nadaraya-Watson kernel-weighted average, then in 2.2 the Robust Locally Weighted Regression and, finally, in 2.3 the proposed approach to select the kernel bandwidth $\lambda$.

\subsection{Nadaraya-Watson kernel method}

Let $\left(\mathbf{x}_{\mathbf{i}}, y_{i}\right)$ denote a response, $y_{i}$, to a recorded value $\mathbf{x}_{\mathbf{i}} \in \mathfrak{R}^{p}$, for $i=1, \ldots, n$. In this paper $\mathbf{x}_{\mathbf{i}}$ denotes the 
environmental variables (inside air temperature, humidity etc.) and the response $y_{i}$ represents the satisfaction degree (real-valued from -3 to +3 ) that the user has given in response to the condition $\mathbf{x}_{\mathbf{i}}$, and then stored in a database. The aim is to assess the response $\hat{y}=\widehat{f(\mathbf{x})}$ (i.e. predict the degree of satisfaction) for any new input value $\mathbf{x}$. The approach aims to estimate a local mean around $\mathbf{x}$, giving all the points in the neighborhood different weights. In particular, we can assign weights that die off smoothly with distance to the target point. In the Nadaraya-Watson kernel method (Bishop, 2006) the resulting estimated response $\hat{y}$ is:

$$
\widehat{f(\mathbf{x})}=\frac{\sum_{i=1}^{n} \mathbf{K}_{\lambda}\left(\mathbf{x}, \mathbf{x}_{\mathbf{i}}\right) y_{i}}{\sum_{i=1}^{n} \mathbf{K}_{\lambda}\left(\mathbf{x}, \mathbf{x}_{\mathbf{i}}\right)}
$$

There are many kernel functions $K_{\lambda}$ proposed in the literature. In this paper we used three different kernel functions among the most used and which generally lead to satisfactory results.

\subsubsection{Epanechnikov quadratic kernel}

The Epanechnikov quadratic kernel is defined as follow:

$$
\mathbf{K}_{\lambda}\left(\mathbf{x}, \mathbf{x}_{\mathbf{i}}\right)=D\left(\frac{\left|\mathbf{x}-\mathbf{x}_{\mathbf{i}}\right|}{\lambda}\right)
$$

with

$$
D(\sigma)= \begin{cases}\frac{3}{4}\left(1-\sigma^{2}\right) & \text { if }|\sigma| \leq 1 \\ 0 & \text { otherwise }\end{cases}
$$

The smoothing parameter $\lambda$, which determines the width of the local neighborhood, has to be chosen. Large $\lambda$ implies lower variance (it takes into account more observation points), but higher bias (it assumes the true function is almost constant inside the window).

\subsubsection{Tricube kernel}

The tricube kernel is another popular compact kernel. It is still defined by Equation (2) but with:

$$
D(\sigma)= \begin{cases}\left(1-|\sigma|^{3}\right)^{3} & \text { if }|\sigma| \leq 1 \\ 0 & \text { otherwise }\end{cases}
$$

This is flatter on the top than Epanechnikov kernel and is differentiable at the boundary.

\subsubsection{Gaussian kernel}

The Gaussian kernel is a popular non-compact kernel. It consists in the Gaussian density function $D(\sigma)=$ $\psi(\sigma)$, which is centered in the query point $\mathbf{x}$, and with the standard deviation playing the role of the window size.

\subsection{Robust locally weighted regression with adaptive bandwidth}

This method is based on the work in (Cleveland, 1979). In the following, we will only describe the proposed LRAB method, while for a more general description of the robust locally weighted regression, the readers should refer to the work in (Cleveland, 1979). Before giving precise details on the LRAB procedure, we attempt to explain the basic idea of the method. Recall that $\left(\mathbf{x}_{\mathbf{i}}, y_{i}\right)$ denotes a response, $y_{i}$, to a recorded value $\mathbf{x}_{\mathbf{i}} \in \mathfrak{R}^{p}$, for $i=1, \ldots, n$, stored in a database.The aim is still to assess the response $\hat{y}=\widehat{f(\mathbf{x})}$ for a new input value $\mathbf{x}$. Let $b(\mathbf{x})$ be a vector of polynomial terms of degree $d$. For example, if we have a linear regression $(d=1)$ in two variables $(p=2)$, we have $b(\mathbf{x})=\left(1, x_{1}, x_{2}\right)$, or if we a quadratic regression $(d=2)$ in two variables $(p=2)$, we have $b(\mathbf{x})=\left(1, x_{1}, x_{1}^{2}, x_{2}, x_{2}^{2}\right)$, At each query point $\mathbf{x} \in \mathfrak{R}^{p}$, the aim is to solve:

$$
\min _{\beta(\mathbf{x})} \sum_{i=1}^{n} \mathbf{K}_{\lambda}\left(\mathbf{x}, \mathbf{x}_{\mathbf{i}}\right)\left(y_{i}-b\left(\mathbf{x}_{\mathbf{i}}\right)^{T} \beta(\mathbf{x})\right)^{2}
$$

to find the estimated function $\widehat{f(\mathbf{x})}=b(\mathbf{x})^{T} \hat{\beta}(\mathbf{x})$. The kernel will be a function such as those defined in section 2.1. This procedure computes the initial fitted values. Anyway, in the real-world, some recorded values $\left(\mathbf{x}_{\mathbf{i}}, y_{i}\right)$ could be affected by noise or be unreliable. The above procedure doesn't itself eliminate such values. The general idea to do this, is to strengthen the above preliminary estimation, assigning a different weight $\psi_{i}$ to each pairs $\left(\mathbf{x}_{\mathbf{i}}, y_{i}\right)$ based on the residual $\left(\hat{y}_{i}-y_{i}\right)$ (the larger the residual, the smaller the associated weight). Then, the equation (3) is computed replacing $\mathbf{K}_{\lambda}$ with $\psi_{i} * \mathbf{K}_{\lambda}$. This is an iterative procedure. In this way, and generally after few iterations, the contribution of some eventually noisy points to the regression is decreased. To achieve this goal, let us define the bisquare function:

$$
\Gamma(\xi)=\left(1-\xi^{2}\right)^{2}
$$

for $|\xi|<1$; otherwise $\Gamma(\xi)=0$. Then, for a fixed new entry point $\mathbf{x}$, let:

$$
\rho_{i}=\left(\hat{y}_{i}-y_{i}\right)
$$



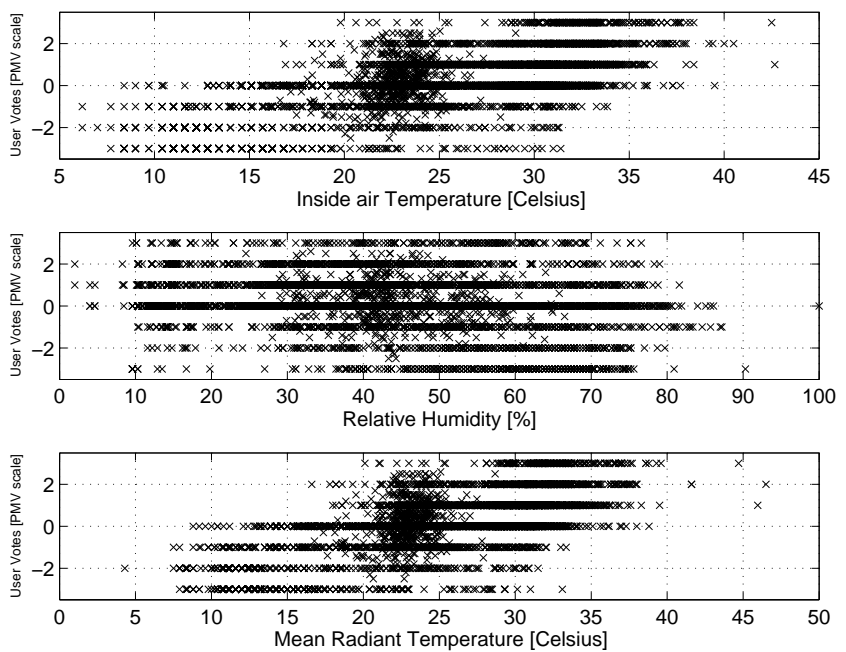

Figure 1: Subset of thermal perceived sensation (in PMV scale) from users vs inside air temperature, humidity and mean radiant temperature from database (ASHRAE, RP884)

be the residuals for $i=1, \ldots, n$, between the original points $y_{i}$ and the estimated points $\hat{y}_{i}$ (i.e. by means of $\hat{\beta}(\mathbf{x})$ ), and let $m$ be the median of the $\left|\rho_{i}\right|$. As described in (Cleveland, 1979), we now choose robustness weights by:

$$
\psi_{i}=\Gamma\left(\rho_{i} / 6 m\right)
$$

At each step of the proposed procedure, the equation (6) is used to update the weight of the kernel function in the equation (3) based on the residual $\rho_{i}$. In this way the value of the kernel function in (3) at each recorded point $\mathbf{x}_{\mathbf{i}}$, is decreased (increased) where the residual value in $\mathbf{x}_{\mathbf{i}}$ (i.e. $\psi_{i}$ ) is too high (too low), so as to improve the regression for the next step.

\subsubsection{Adaptive bandwidth}

In order to choose the bandwidth $\lambda$, we first needs to take into account the fact that the density of the recorded data may be variable. Figure 1 shows a global view of a subset of field data from a public database (ASHRAE, RP-884), which were used for our tests. In this figure, for all three graphs, it shows the thermal perception from users (reported with the PMV scale) in respect to three environmental variables, i.e. inside air temperature, humidity and mean radiant temperature (MRT).

As shown in fig. 1, there are areas in which the data are clustered closely together (in the center), while, on other hand, other areas are characterised by sparse data (on the boundaries). If in this situation we use a fixed value for the bandwidth $\lambda$, the result is what is shown, as an example, in figures 3

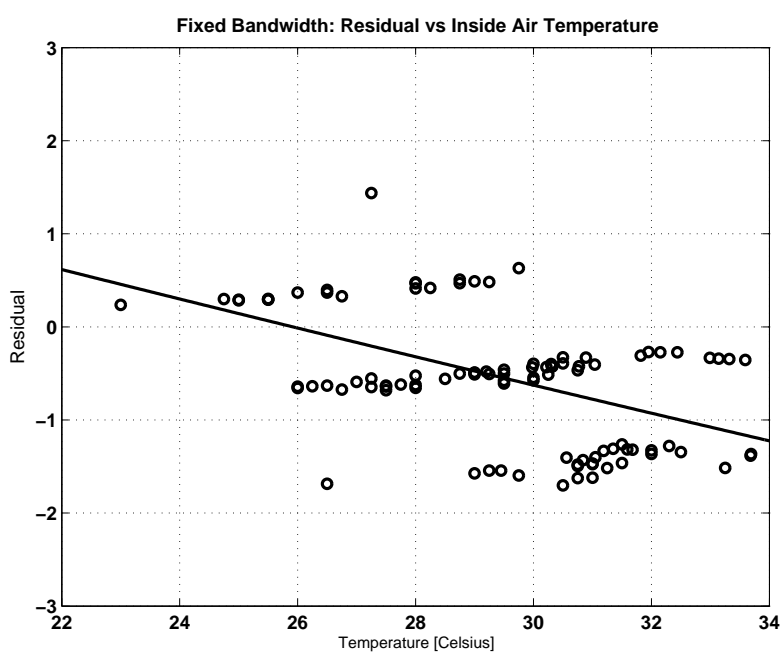

Figure 2: Residual vs inside air temperature, for an user with 100 records from (ASHRAE, RP-884) using a regression in 2 variables with a fixed bandwidth $\lambda$ for the kernel

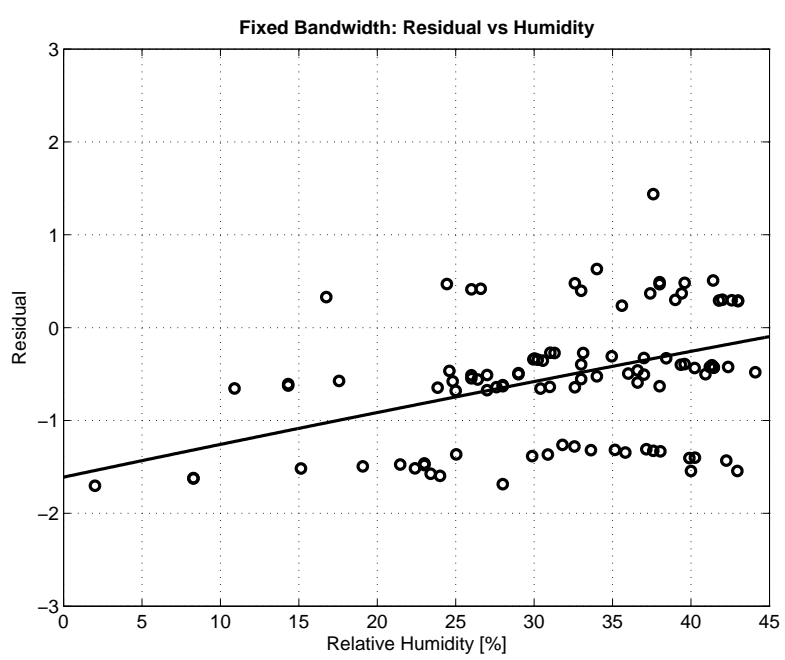

Figure 3: Residual vs humidity, for an user with 100 records from (ASHRAE, RP-884) using a regression in 2 variables with a fixed bandwidth $\lambda$ for the kernel

and 4. In these figures, we report the residual (i.e. the difference between the predicted vote and the actual vote) computing a regression with $p=2$ (using inside air temperature and humidity) for a random user with 100 votes stored. It easy to see that the residual tends to vary with the values of the input variables, and this is generally an undesired effect for any kind of predicting technique. That effect occurs because, using a fixed bandwidth there are less points supporting the regression where the data are sparse and, on other hand, more supporting points where the data are denser.

In view of this, it would be appropriate to have a large smoothing parameter where the data are sparse, 


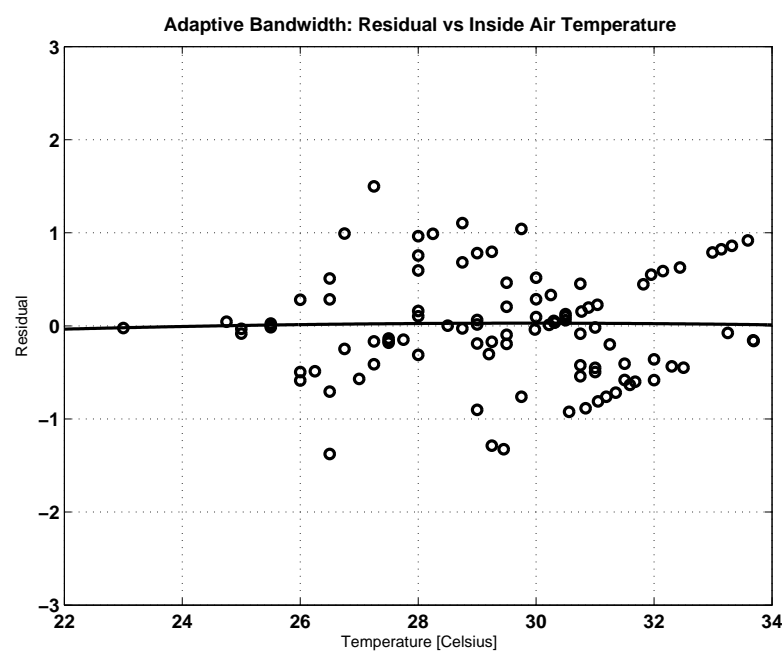

Figure 4: Residual vs inside air temperature, for the user in figures 2 and 3 using a regression in 2 variables with an adaptive bandwidth $\lambda$ for the kernel

and a smaller smoothing parameter where the data are denser. In this situation an adaptive parameter has been introduced. Let the ratio $\mathrm{v} / n$ (where $\mathrm{v}<n$ ), describe the proportion of the sample which contributes strictly positive weight to each local regression (for example if the ratio is 0.7 , it means that $70 \%$ of the recorded data contributes to the regression). Once we have chosen $\mathrm{v} / n$ (that means we have chosen $\mathrm{v}$, as $n$ is fixed), we select the $v$ nearest neighbours from the new entry point $\mathbf{x}$. Then, the smoothing parameter $\lambda$ is denoted by the distance of the most distant neighbour among the $v$ neighbours selected. It should be noted that the entire procedure requires the choice of a single parameter setting. This choice generally improves the previous effect, giving rise to a results as those shown in the figures 4 and 5, where the residual is kept more constant for both input variables.

\subsubsection{The algorithm}

The proposed method can be described by the following sequence of operations (table 1): the algorithm is initialized by setting only one parameter (step 1). Then, for each new entry point $\mathbf{x}$ (step 2), it first computes an initial fitting (step 2.1), then it strengthens the initial regression by the steps 2.2 .1 to 2.2 .2 , performing the sub-procedure described in the previous section, iteratively. If we have $M$ new entry points $\mathbf{x}$ in total, the steps from 2.1 to 2.3 are repeated $M$ times (one time for each new entry point).

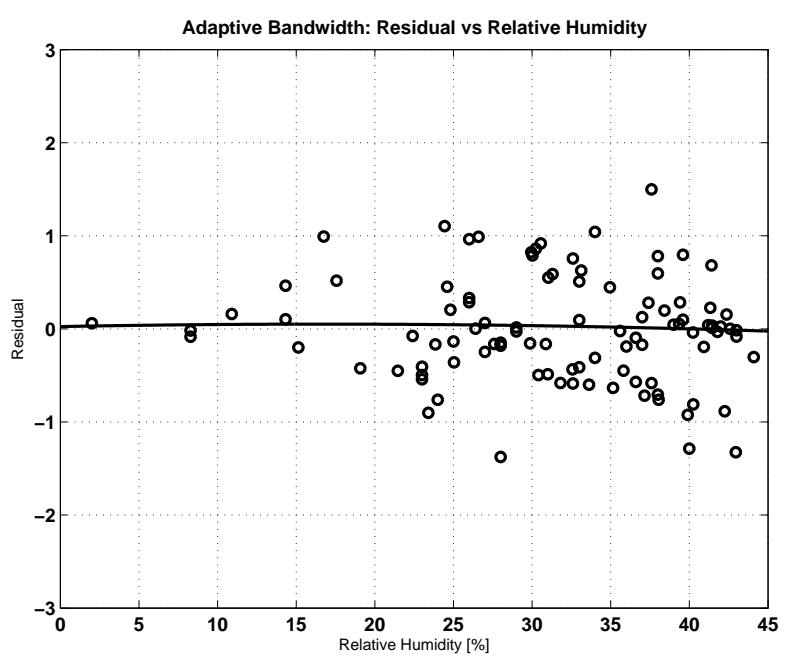

Figure 5: Residual vs humidity, for the user in figures 2 and 3 using a regression in 2 variables with an adaptive bandwidth $\lambda$ for the kernel

\section{LRAB}

1: Initialize: set parameters $v$

2: For each entry point $x$ :

2.1: $\quad$ minimise (3)

2.2: $\quad$ while iterations $<\max$ iterations do:

2.2.1: $\quad$ for each $i$ compute (6)

2.2.2: $\quad$ minimise (3) replacing $\mathbf{K}_{\lambda}$ with $\psi_{i} * \mathbf{K}_{\lambda}$

2.3: end while

3: end

Table 1: Pseudocode for the proposed LRAB

\section{EXPERIMENTS}

This section describes the experimental results obtained from a comparison between the proposed method and both the Nadaraya-Watson kernel method and the PMV method. The three kernel functions described in section 2.1 are used to compute and compare LRAB and Nadaraya-Watson methods. It should be noted that, although PMV is not based on a learning approach, in this paper, we compare our method with PMV since the latter is the international standard used to predict comfort in current building design and operation (ISO, 1994).

In particular, LRAB has been compared with the aforementioned methods on real data from the ASHRAE RP-884 database. This collection contains 52 studies with more than 20,000 user comfort votes from different climate zones. However, some of these 
field studies contain only a few votes for each user. Thus they are not well suited for testing the proposed algorithm. This is because our approach seeks to learn the user preferences based on their votes, and it requires sufficiently many data records. For this reason, only the users with more than 10 votes have been used to compute the proposed LRAB. After removing the studies and records as described above we were left with 5 climate zones, 161 users and 6421 records.

LRAB has been implemented in Matlab ${ }^{\mathrm{TM}}$, using the trust-region method to minimize the problem in (3), with a termination tolerance of $10^{-6}$. The experiments have been performed through leave-one-out validation, for each user (i.e. using a single observation from the original sample as the validation data, and the remaining observations as the training data).

The algorithms are evaluated considering the difference $|\delta V|$ between the computed votes (by all methods) and the actual vote (reported in the database). As with the field studies (Ari et al., 2008),(Schumann et al., 2010), a prediction is defined to be accurate if $|\delta V|<0.7$.

The computation time depends on the number of points have to be evaluated for each prediction; for example, we've found that for predictions involving 100 records they take, on average, less than 5 seconds.

In the following tables 2, 3 and 4 are reported the comparison (in terms of percentage of success) between LRAB, Nadaraya-Watson and PMV using respectively one, two and three variables.

Table 2: Comparison between LRAB, Nadaraya-Watson and PMV using only one variable for LRAB and NadarayaWatson, giving percentage accuracies.

\begin{tabular}{lccc}
\hline & Temperature & MRT & Humidity \\
\hline PMV & \multicolumn{3}{c}{38.47} \\
\hline \multicolumn{4}{c}{ LRAB } \\
\hline Tricube & 64.69 & 64.42 & 57.78 \\
Epanechnikov & 64.44 & 64.34 & 55.91 \\
Gaussian & 60.06 & 61.11 & 51.90 \\
\hline \multicolumn{4}{c}{ Nadaraya-Watson } \\
\hline Tricube & 49.48 & 50.19 & 40.11 \\
Epanechnikov & 52.07 & 53.38 & 41.89 \\
Gaussian & 51.61 & 52.21 & 35.55 \\
\hline
\end{tabular}

Tables 2, 3 and 4 illustrate how accurately the LRAB predicts the actual comfort vote of each user compared with Nadaraya-Watson and PMV. With
Table 3: Comparison between LRAB, Nadaraya-Watson and PMV using two variables for LRAB and NadarayaWatson, giving percentage accuracies.

\begin{tabular}{lcc}
\hline & Temp. and Hum. & Temp. and MRT \\
\hline PMV & \multicolumn{2}{c}{38.47} \\
\hline \multicolumn{3}{c}{ LRAB } \\
\hline Tricube & 66.70 & 67.03 \\
Epanechnikov & 65.91 & 65.84 \\
Gaussian & 65.70 & 65.14 \\
\hline \multicolumn{3}{c}{ Nadaraya-Watson } \\
\hline Tricube & 39.81 & 50.87 \\
Epanechnikov & 41.12 & 50.22 \\
Gaussian & 38.22 & 49.81 \\
\hline
\end{tabular}

Table 4: Comparison between LRAB, Nadaraya-Watson and PMV using three variables for LRAB and NadarayaWatson, giving percentage accuracies.

\begin{tabular}{lc}
\hline & Temp., Hum and MRT \\
\hline PMV & 38.47 \\
\hline \multicolumn{2}{c}{ LRAB } \\
\hline Tricube & 69.27 \\
Epanechnikov & 69.22 \\
Gaussian & 65.01 \\
\hline \multicolumn{2}{c}{ Nadaraya-Watson } \\
\hline Tricube & 45.17 \\
Epanechnikov & 43.63 \\
Gaussian & 50.19 \\
\hline
\end{tabular}

only one variable, LRAB achieves more than $64 \%$ accuracy (using both tricube and Epanechnikov kernels), while PMV only achieves 38\% accuracy. The other considered kernel method, the NadarayaWatson method with $52-53 \%$ accuracy also improves on the PMV method, but doesn't match the accuracy of the proposed LRAB method. Similarly results are shown in Tables 3 and 4 for respectively two and three variables. In particular, the best result is achieved with three variables where LRAB achieves $69 \%$ accuracy with both tricube and Epanechnikov kernels.

Moreover, one can argue that kernel methods, un- 


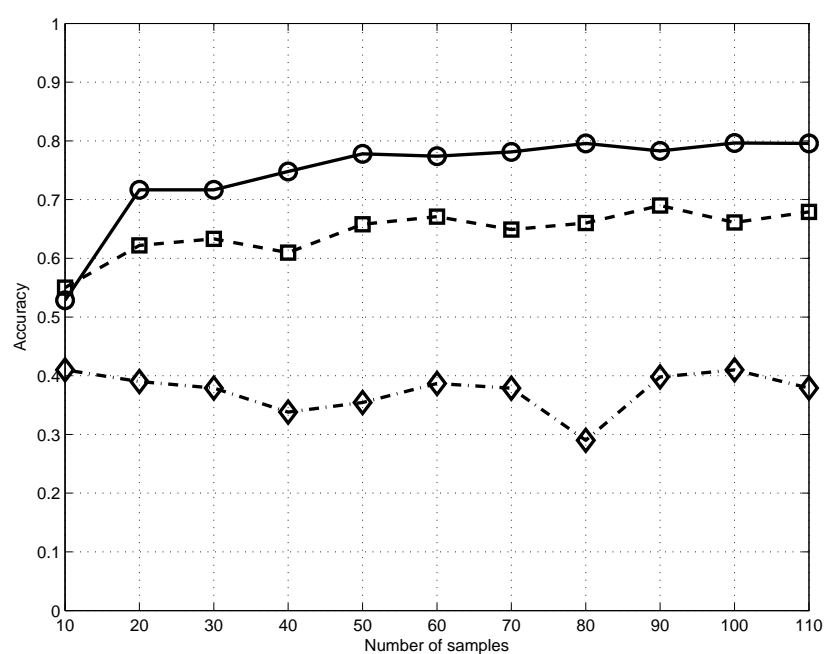

Figure 6: Accuracy vs number of samples using 3 variables for: LRAB (circle dots), Nadaraya-Watson (square dots) and PMV (diamond dots).

like PMV, are essentially methods that learn from the data, and so they require a sufficient amount of data to give the best results. To test the influence of the amount of data on the proposed LRAB, we've statistically investigated this effect on a subset of users from the database (ASHRAE, RP-884). This subset includes 39 users, with at least 110 comfort votes from each single user. We investigated the variation of the percentage of success (according to the accuracy level $|\delta V|<0.7)$ of the LRAB and Nadaraya-Watson using three input variables, and the PMV, varying the number of samples used for the above methods in a range from 10 to 110 samples. The results are shown in figure 6 , showing that from 50 to 110 samples, the accuracy of the LRAB ( $80 \%$ in this case) remains roughly constant. Hence, based on this investigation, we can conclude that the level of accuracy certainly is biased by the amount of data used in the regression, but this is true especially for a small amount of data, and the results do not show much improvement after a certain threshold. Finally, we can also see that LRAB overcomes the Nadaraya - Watson method for almost the entire tested range (from 20 up to 110 samples).

\section{CONCLUSIONS}

In the present paper, we have proposed the use of robust locally weighted regression with an adaptive bandwidth to predict individual thermal comfort. The approach has been characterized and compared with an other standard kernel method (i.e. the Nadaraya-Watson method) and with the traditional
PMV approach. The experiments were carried out using publicly available datasets: they have shown that our LRAB outperforms both Nadaraya-Watson and the traditional PMV approaches in predicting thermal comfort. We also investigated the influence of the amount of the data used by LRAB on its performance, concluding that the performance only degrades when the predicted value is based on just a small number of samples (less than 40 in this case). Since LRAB can be computed quickly, and requires only a single parameter setting that is easily obtained, then if individual comfort responses are available, this method is feasible for use as a comfort measure in real time control. The next step will the extension of the present work to a similar and more challenging context: balancing the preferences of a number of different occupants sharing the same space in buildings (shared offices)(Wilson, 2012).

This work is part of the Strategic Research Cluster project ITOBO (supported by the Science Foundation Ireland), for which we are acquiring occupant comfort reports and fine grained sensor data, and constructing validated physical models of the building and its HVAC systems. The intention is then to use the comfort reports and sensor data as input to our LRAB method, and then to use the output of LRAB as the input to intelligent control systems which optimise the internal comfort for the specific individual occupants.

\section{ACKNOWLEDGEMENTS}

This work is supported by Intel Labs Europe and IRCSET through the Enterprise Partnership Scheme, and also in part by Science Foundation Ireland through SFI Research Cluster ITOBO (grant No. 07.SRC.I1170), and grant No. 08/PI/I1912.

\section{REFERENCES}

Ari, S., Wilcoxen, P., Khalifa, H., Dannenhoffer, J., and Isik, C. (2008). A practical approach to individual thermal comfort and energy optimization problem. In Fuzzy Information Processing Society, 2008. NAFIPS 2008. Annual Meeting of the North American, pages 1 -6 .

ASHRAE (2010). ASHRAE Standard: Thermal Environmental Conditions for Human Occupancy. ASHRAE.

Atthajariyakul, S. and Leephakpreeda, T. (2005). Neural computing thermal comfort index for HVAC systems.

Bingxin, M., Jiong, S., and Yanchao, W. (2011). Experimental design and the GA-BP prediction of human thermal comfort index. In Natural Computation 
(ICNC), 2011 Seventh International Conference on, volume 2, pages $771-775$.

Bishop, C. M. (2006). Pattern Recognition and Machine Learning.

Busch, J. F. (1992). A tale of two populations: thermal comfort in air-conditioned and naturally ventilated offices in Thailand. Energy and Buildings, 18(3-4):235 -249 .

Cleveland, W. S. (1979). Robust locally weighted regression and smoothing scatterplots. Journal of the American Statistical Association, 74(368):pp. 829-836.

Cleveland, W. S. and Devlin, S. J. (1988). Locally weighted regression: An approach to regression analysis by local fitting. Journal of the American Statistical Association, 83(403):pp. 596-610.

Daum, D., Haldi, F., and Morel, N. (2011). A personalized measure of thermal comfort for building controls. Building and Environment, 46(1):3-11.

de Dear, R. and Schiller Brager, G. (2001). The adaptive model of thermal comfort and energy conservation in the built environment. International Journal of Biometeorology, 45:100-108.

de Dear, R. J. and Brager, G. S. (2002). Thermal comfort in naturally ventilated buildings: revisions to ashrae standard 55. Energy and Buildings, 34(6):549 - 561.

Fanger, P. (1972). Thermal comfort: analysis and applications in environmental engineering. McGraw-Hill, New York

Federspiel, C. C. and Asada, H. (1992). User-adaptable comfort control for HVAC systems. In American Control Conference, 1992, pages 2312 -2319.

Feldmeier, M. and Paradiso, J. A. (2010). Personalized hvac control system. In In Internet of Things 2010 Conference.

Freire, R. Z., Oliveira, G. H., and Mendes, N. (2008). Predictive controllers for thermal comfort optimization and energy savings. Energy and Buildings, 40(7):1353 -1365 .

Gagge, A. P., Fobelets, A. P., and Berglund, L. G. (1986). A standard predictive index of human response to the thermal environment.

Hastie, T., Tibshirani, R., and Friedman, J. H. (2003). The Elements of Statistical Learning. Springer, corrected edition.

Humphreys, M. and Nicol, J. (2000). Effects of measurement and formulation error on thermal comfort indices in the ashrae database of field studies. ASHRAE transactions, 106:493-502.

Humphreys, M. A. and Nicol, J. F. (2002). The validity of iso-pmv for predicting comfort votes in everyday thermal environments. Energy and Buildings, 34(6):667 - 684

ISO (1994). ISO 7730: Moderate Thermal EnvironmentsDetermination of the PMV and PPD Indices and Specification of the Conditions for Thermal Comfort. ISO.

Kumar, S. and Mahdavi, A. (2001). Integrating thermal comfort field data analysis in a case-based building simulation environment. Building and Environment, 36(6):711 - 720
Olesen, B. W. (2004). International standards for the indoor environment. Indoor Air, 14:18-26.

Schumann, A., Wilson, N., and Burillo, M. (2010). Learning user preferences to maximise occupant comfort in office buildings. In Proceedings of the 23rd international conference on Industrial engineering and other applications of applied intelligent systems - Volume Part I, IEA/AIE'10, pages 681-690, Berlin, Heidelberg. Springer-Verlag.

Sherman, M. (1985). A simplified model of thermal comfort. Energy and Buildings, 8(1):37 - 50 .

Shukor, S. A. A., Kohlhof, K., and Jamal, Z. A. Z. (2007). Development of a pmv-based thermal comfort modelling. In Proceedings of the 18th IASTED International Conference: modelling and simulation, MOAS'07, pages 670-675, Anaheim, CA, USA. ACTA Press.

Wilson, N. (2012). On balancing occupants' comfort in shared spaces. In Proc. 6th Multidisciplinary Workshop on Advances in Preference Handling (MPREF12).

Yang, K. and Su, C. (1997). An approach to building energy savings using the PMV index. Building and Environment, 32(1):25 - 30 .

Yang, W. and Zhang, G. (2008). Thermal comfort in naturally ventilated and air-conditioned buildings in humid subtropical climate zone in china. International Journal of Biometeorology, 52:385-398. 